\title{
Classification of Zero Divisor Graphs of a Commutative Ring With Degree Equal 7 and 8
}

Nazar H. Shuker

nazarh_2013@yahoo.com

Husam Q. Mohammad

College of Computer Sciences and Mathematics

husam_alsabawi@yahoo.com

University of Mosul, Mosul, Iraq

Received on: 15/05/2012

\section{ABSTRACT}

Accepted on: 18/09/2012

In $2005 \mathrm{~J}$. T Wang investigated the zero divisor graphs of degrees 5 and 6 . In this paper, we consider the zero divisor graphs of a commutative rings of degrees 7 and 8 .

Keywords: Zero-divisor, Ring, Zero-divisor graph.

$$
\begin{aligned}
& \text { تصنيف بيانات قواسم الصفر للحلقات الإبدالية ذات الارجات } 7 \text { و } 8
\end{aligned}
$$

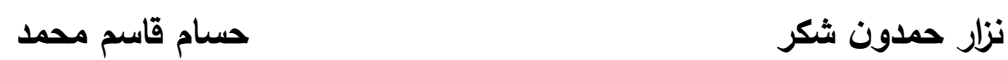

$$
\begin{aligned}
& \text { كلية علوم الحاسوب والرياضيات، جامعة الموصل } \\
& \text { تايخ استلام البحث: 2012/05/15 2012/09/18 } \\
& \text { الملخص } \\
& \text { في عام } 2005 \text { درس Wang بيانات قواسم الصفر للحلقات الإبدالية من الدرجة } 5 \text { و6. في هذا البحث } \\
& \text { درسنا بيانات قواسم الصفر للحلقات الإبدالية من الدرجتين } 7 \text { و } 8 . \\
& \text { الكلمات المفتاحية: قواسم الصفر، حلقة، بيان قواسم الصفر . }
\end{aligned}
$$

\section{Introduction}

The concept of zero divisor graph of a commutative ring was introduced by Beck in [3]. He let all the elements of the ring be vertices of the graph. In [1] Anderson and Livingston introduced and studied the zero divisor graph whose vertices are the non-zero zero divisors.

Throughout this paper, all rings are assumed to be commutative rings with identity, and $Z(R)$ be the set of zero divisors. We associate a simple graph $\Gamma(R)$ to a ring $R$ with vertices $Z(R)^{*}=Z(R)-\{0\}$, the set of all non-zero zero divisors of $R$. For all distinct $x, y \in Z(R)^{*}$, the vertices $x$ and $y$ are adjacent if and only if $x y=0$. $(R, m)$ and $|S|$ will stand respectively for the local ring with maximal ideal $\mathrm{m}$ and cardinal numbers of a set $\mathrm{S}$. connected.

In [1] Anderson and Livingston proved that for any commutative ring $R \Gamma(R)$ is

In 2005 J. T Wang [5] investigated the zero divisor graphs of degrees 5 and 6. In this paper, we extend this results to consider the zero divisor graphs of commutative rings of degrees 7 and 8 .

The main result when $\left|\mathrm{Z}(\mathrm{R})^{*}\right|=7$ is given in Theorem 2.7 , while when $\left|\mathrm{Z}(\mathrm{R})^{*}\right|=8$ the main result is given in Theorem 3.4. We also extend Wang's result concerning local rings (Theorem 2.2)

\section{Rings with $\mid \mathrm{Z}(\mathbf{R}) *=7$}

It is known that if $\mathrm{R}$ is a ring then $\Gamma(\mathrm{R})$ is connected. In this section, we find all possible graphs of $\Gamma(\mathrm{R})$ with $\Gamma(\mathrm{R})=7$.

Recall that if $\mathrm{R}$ is finite ring, then every element of $\mathrm{R}$ is either a unit or a zero divisor [2]. In [5] Wang proved the following result. 


\section{Lemma 2.1 :} $\left|\mathrm{m}_{1}\right|\left|\mathrm{m}_{2}\right|-1$.

Let $\left(\mathrm{R}_{1}, \mathrm{~m}_{1}\right)$ and $\left(\mathrm{R}_{2}, \mathrm{~m}_{2}\right)$ are local rings, then $\left|\mathrm{Z}\left(\mathrm{R}_{1} \mathrm{x} \mathrm{R}_{2}\right)^{*}\right|=\left|\mathrm{R}_{1}\right| \mathrm{x}\left|\mathrm{m}_{2}\right|+\left|\mathrm{R}_{2}\right| \mathrm{x}\left|\mathrm{m}_{1}\right|^{-}$

Now, we shall prove the following theorem which extends Wang's result.

\section{Theorem 2.2 :}

If $\left(\mathrm{R}_{1}, \mathrm{~m}_{1}\right), \quad\left(\mathrm{R}_{2}, \mathrm{~m}_{2}\right)$ and $\left(\mathrm{R}_{3}, \mathrm{~m}_{3}\right)$ are finite local rings, then $\left|\mathrm{Z}\left(\mathrm{R}_{1} \times \mathrm{R}_{2} \times \mathrm{R}_{3}\right)^{*}\right|=\left|\mathrm{R}_{1}\right| \mathrm{x}\left|\mathrm{R}_{2}\right| \mathrm{x}\left|\mathrm{m}_{3}\right|+\left|\mathrm{Z}\left(\mathrm{R}_{1} \times \mathrm{R}_{2}\right)\right| \mathrm{x}\left(\left|\mathrm{R}_{3}\right|-\left|\mathrm{m}_{3}\right|\right)-1$ where $\left|\mathrm{Z}\left(\mathrm{R}_{1} \mathrm{xR}_{2}\right)\right|=\left|\mathrm{R}_{1}\right| \mathrm{x}\left|\mathrm{m}_{2}\right|+\left|\mathrm{R}_{2}\right| \mathrm{x}\left|\mathrm{m}_{1}\right|-\left|\mathrm{m}_{1}\right| \mathrm{x}\left|\mathrm{m}_{2}\right|$.

\section{Proof :}

By Lemma 2.1 $\left|\mathrm{Z}\left(\mathrm{R}_{1} \mathrm{xR}_{2}\right)^{*}\right|=\left|\mathrm{R}_{1}\right| \mathrm{x}\left|\mathrm{m}_{2}\right|+\left|\mathrm{R}_{2}\right| \mathrm{x}\left|\mathrm{m}_{1}\right|-\left|\mathrm{m}_{1}\right| \mathrm{x}\left|\mathrm{m}_{2}\right|-1$, therefore $\left|\mathrm{Z}\left(\mathrm{R}_{1} \mathrm{xR}_{2}\right)\right|=\left|\mathrm{Z}\left(\mathrm{R}_{1} \mathrm{xR}_{2}\right)^{*}\right|+1=\left|\mathrm{R}_{1}\right| \mathrm{x}\left|\mathrm{m}_{2}\right|+\left|\mathrm{R}_{2}\right| \mathrm{x}\left|\mathrm{m}_{1}\right|-\left|\mathrm{m}_{1}\right| \mathrm{x}\left|\mathrm{m}_{2}\right|$. Let $\mathrm{R}_{(1)(2)}=\mathrm{R}_{1} \mathrm{xR}_{2}$, then $\left|R_{(1)(2)}\right|=\left|R_{1}\right| x\left|R_{2}\right|$ and $\left|Z\left(R_{(1)(2)}\right)\right|=Z\left(R_{1} \times R_{2}\right)$.

For any non-zero-divisor $(a, b)$ in $R_{(1)(2)} \times R_{3}$, we have the following cases:

1 - If $\mathrm{a}$ is non-zero divisor of $\mathrm{R}_{(1)(2)}$, then a must be a unit element. If $\mathrm{b}$ is a zero divisor of $R_{3}$, then there are $\left(\left|R_{(1)(2)}\right|-\left|Z\left(R_{(1)(2)}\right)\right|\right) x\left|m_{3}\right|$ elements of this type.

2- If $\mathrm{a}$ is a non-zero zero divisor of $\mathrm{R}_{(1)(2)}$ and $\mathrm{b}$ any element in $\mathrm{R}_{3}$, then there are $\left(\left|\mathrm{Z}\left(\mathrm{R}_{(1)(2))}\right)\right|-1\right) \mathrm{x}\left|\mathrm{R}_{3}\right|$ elements of this type.

3- If $a=0$, and $b$ is a non-zero element in $R_{3}$, then there are $1 x\left(\left|R_{3}\right|-1\right)$.

Now, we sum up these three types of elements; there are as follows:

$\left(\left|\mathrm{R}_{(1)(2)}\right|-\left|\mathrm{Z}\left(\mathrm{R}_{(1)(2)}\right)\right|\right) \mathrm{x}\left|\mathrm{m}_{3}\right|+\left(\left|\mathrm{Z}\left(\mathrm{R}_{(1)(2))}\right)\right|-1\right) \mathrm{x}\left|\mathrm{R}_{3}\right|+1 \mathrm{x}\left(\left|\mathrm{R}_{3}\right|-1\right)=$

$\left|\mathrm{R}_{(1)(2)}\right| \mathrm{x}\left|\mathrm{m}_{3}\right|-\left|\mathrm{Z}\left(\mathrm{R}_{(1)(2)}\right)\right| \mathrm{x}\left|\mathrm{m}_{3}\right|+\left|\mathrm{Z}\left(\mathrm{R}_{(1)(2)}\right)\right| \mathrm{x}\left|\mathrm{R}_{3}\right|-\left|\mathrm{R}_{3}\right|+\left|\mathrm{R}_{3}\right|-1=$

$\left|R_{1}\right| x\left|R_{2}\right| x\left|m_{3}\right|+\left|Z\left(R_{1} \times R_{2}\right)\right|\left(\left|R_{3}\right|-\left|m_{3}\right|\right)-1$ where

$\mathrm{Z}\left(\mathrm{R}_{1} \mathrm{xR}_{2}\right)|=| \mathrm{R}_{1}|\mathrm{x}| \mathrm{m}_{2}|+| \mathrm{R}_{2}|\mathrm{x}| \mathrm{m}_{1}|-| \mathrm{m}_{1}|\mathrm{x}| \mathrm{m}_{2} \mid$.

As a direct consequence to Theorem 2.2, we obtain the following:

\section{Corollary 2.3 :}

If $R_{1}, R_{2}$ and $R_{3}$ are finite fields, then

$\left|\mathrm{Z}\left(\mathrm{R}_{1} \mathrm{xR}_{2} \mathrm{XR}_{3}\right)^{*}\right|=\left|\mathrm{R}_{1}\right|\left|\mathrm{R}_{2}\right|+\left|\mathrm{R}_{1}\right|\left|\mathrm{R}_{3}\right|+\left|\mathrm{R}_{2}\right|\left|\mathrm{R}_{3}\right|-\left|\mathrm{R}_{1}\right|-\left|\mathrm{R}_{2}\right|-\left|\mathrm{R}_{3}\right|$.

\section{Corollary 2.4 :} field.

If $\mathrm{R}$ finite and $\mathrm{R} \cong \mathrm{R}_{1} \times \mathrm{R}_{2} \times \mathrm{R}_{3}$, then $\left|\mathrm{Z}(\mathrm{R})^{*}\right| \geq 13$ for some local rings $\mathrm{R}_{\mathrm{i}}$ but not

\section{Proof :}

Suppose that $R_{3}$ is local which is not a field, then clearly $\left|R_{3}\right| \geq 4$ and $\left|m_{3}\right| \geq 2$ and since $\left|R_{1}\right|,\left|R_{2}\right| \geq 2$ and $\left|m_{1}\right|,\left|m_{2}\right| \geq 1$, then $Z\left(R_{1} \times R_{2}\right) \geq 3$, therefore $|Z(R) *| \geq 2.2 .2+3(4-2)-1=13$.

Next, we prove two fundamental lemmas

\section{Lemma 2.5 :}

If $\mathrm{R}$ is a ring with $|\mathrm{Z}(\mathrm{R})|^{*}=7$, then is either $\mathrm{R}$ local ring or $\mathrm{R}$ is isomorphic to a product of two local rings.

\section{Proof:}

Since $\left|\mathrm{Z}(\mathrm{R})^{*}\right|=7$, then $\mathrm{R}$ is finite and hence $\mathrm{R} \cong \mathrm{R}_{1} \times \mathrm{R}_{2} \mathrm{x} \ldots \mathrm{R}_{\mathrm{n}}$ where $\mathrm{R}_{\mathrm{i}}$, $\mathrm{i}=1,2, \ldots, \mathrm{n}$ are local rings. If $\mathrm{n} \geq 4$, then by [5,Lemma 4.7], $\left|\mathrm{Z}(\mathrm{R})^{*}\right| \geq 14$ this is a contradiction.

Now, consider $n=3$, if $R_{i}$ local, but not field for some $1 \leq i \leq 3$, then by Corollary 2.4, $\left|\mathrm{Z}(\mathrm{R})^{*}\right| \geq 13$ which is a contradiction. Hence $\mathrm{R}_{\mathrm{i}}$ are fields for all $1 \leq \mathrm{i} \leq 3$. Applying Corollary $2.3\left|\mathrm{Z}\left(\mathrm{R}_{1} \times \mathrm{R}_{2} \times \mathrm{R}_{3}\right)^{*}\right|=\left|\mathrm{R}_{1}\right|\left|\mathrm{R}_{2}\right|+\left|\mathrm{R}_{1}\right|\left|\mathrm{R}_{3}\right|+\left|\mathrm{R}_{2}\right|\left|\mathrm{R}_{3}\right|-\left|\mathrm{R}_{1}\right|-\left|\mathrm{R}_{2}\right|-\left|\mathrm{R}_{3}\right|=7$. If $\left|\mathrm{R}_{1}\right|=\left|\mathrm{R}_{2}\right|=2$ 
then $\left|R_{3}\right|=7 / 3$ which is also a contradiction. Finally, if $\left|R_{i}\right| \geq 3$ for some $i$, then by [5,Lemma 4.5], $\left|\mathrm{Z}(\mathrm{R})^{*}\right| \geq 9$ which is also a contradiction. Therefore, $\mathrm{n}=1$ or 2

\section{Lemma 2.6 :}

Let $\mathrm{R}$ be a ring which is not local and $|\mathrm{Z}(\mathrm{R})|^{*}=7$, then $\mathrm{R} \cong \mathrm{Z}_{4} \mathrm{XZ} \mathrm{Z}_{3}$ or $\mathrm{Z}_{2}[\mathrm{X}] /\left(\mathrm{X}^{2}\right) \mathrm{x}$ $\mathrm{Z}_{3}$ or $\mathrm{Z}_{2} \mathrm{XZ}_{7}$ or $\mathrm{F}_{4} \mathrm{x} \mathrm{Z}_{5}$.

\section{Proof:}

Suppose that $R$ is a ring which is not local, then by Lemma $2.5 R \cong R_{1} \times R_{2}$. If $R_{1}$ and $R_{2}$ are local, but not a field, then by [5, Corollary 4.4], $\left|\mathrm{Z}(\mathrm{R})^{*}\right| \geq 11$ which is a contradiction. If $R_{1}$ local, but not a field, $R_{2}$ field, then we have $\left|\mathrm{Z}(\mathrm{R})^{*}\right|=\left|\mathrm{R}_{1}\right| \mathrm{x}\left|\mathrm{m}_{2}\right|+\left|\mathrm{R}_{2}\right| \mathrm{x}\left|\mathrm{m}_{1}\right|-\left|\mathrm{m}_{1}\right| \mathrm{x}\left|\mathrm{m}_{2}\right|-1=7$, this yields to $\left|\mathrm{R}_{1}\right|+\left|\mathrm{m}_{1}\right|\left(\left|\mathrm{R}_{2}\right|-1\right)-8=0 \ldots$ (1)

Now, if $\left|\mathrm{m}_{1}\right|=\mathrm{p}$ where $\mathrm{p}$ is prime number, then by [5, Lemma 4.2], $\left|\mathrm{R}_{1}\right|=\left|\mathrm{m}_{1}\right|^{*}=\mathrm{p}^{2}$, so from equation (1) we have $\mathrm{p}^{2}+\mathrm{kp}-8=0 \quad \ldots(2)$, where $\mathrm{k}=\left|\mathrm{R}_{2}\right|-1$ this implies that $p=\frac{-k+\sqrt{k^{2}+32}}{2}$, so the only solution for $\mathrm{p}$ to be prime is $\mathrm{k}=2$, and hence $\mathrm{p}=2$, and this implies $\left|R_{1}\right|=4$ and $\left|R_{2}\right|=3$. Then, by [4,pp.687] $R_{1} \cong Z_{4}$ or $Z_{2}[X] /\left(X^{2}\right)$ and $R_{2} \cong Z_{3}$. Hence, $\mathrm{R} \cong \mathrm{Z}_{4} \mathrm{XZ} \mathrm{Z}_{3}$ or $\mathrm{Z}_{2}[\mathrm{X}] /\left(\mathrm{X}^{2}\right) \times \mathrm{Z}_{3}$. Now if $\mathrm{R}_{1}$ and $\mathrm{R}_{2}$ are fields, then $\left|\mathrm{Z}(\mathrm{R})^{*}\right|=\left|\mathrm{R}_{1}\right|+\left|\mathrm{R}_{2}\right|-$ $2=7$, this yields to $\left|R_{1}\right|+\left|R_{2}\right|=9$. Therefore, $\left|R_{1}\right|=2,\left|R_{2}\right|=7$ or $\left|R_{1}\right|=4,\left|R_{2}\right|=5$. Thus, $\mathrm{R} \cong \mathrm{Z}_{2} \mathrm{x} \mathrm{Z}_{7}$ or $\mathrm{F}_{4} \mathrm{x} \mathrm{Z}_{5}$.

Now, we shall prove the main result of this section.

\section{Theorem 2.7 :}

Let $\mathrm{R}$ be a ring which is not local and $|\mathrm{Z}(\mathrm{R})|^{*}=7$, then the following graph can be realized as $\Gamma(\mathrm{R})$

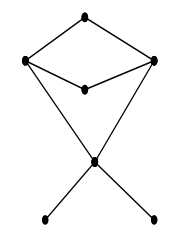

Figure (1)

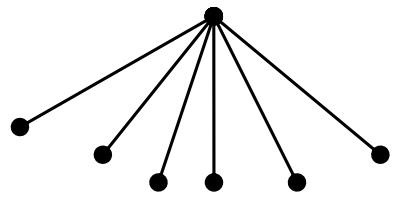

Figure (2)

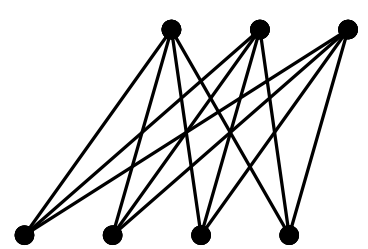

Figure (3),

\section{Proof:}

By Lemma $2.6, \mathrm{R} \cong \mathrm{Z}_{4} \mathrm{x} \mathrm{Z}_{3}$ or $\mathrm{Z}_{2}[\mathrm{X}] /\left(\mathrm{X}^{2}\right) \mathrm{x} \mathrm{Z}_{3}$ or $\mathrm{Z}_{2} \mathrm{xZ}_{7}$ or $\mathrm{F}_{4} \mathrm{x} \mathrm{Z}_{5}$. In Figure (1), can be realized as $\Gamma\left(Z_{4} x Z_{3}\right)$ or $\Gamma\left(Z_{2}[X] /\left(X^{2}\right) x Z_{3}\right)$, Figure (2) can be realized as $\Gamma\left(Z_{2} x Z_{7}\right)$ and Figure (3) can be realized as $\Gamma\left(\mathrm{F}_{4} \times Z_{5}\right)$.

\section{Rings with $\left|\mathrm{Z}(\mathbf{R})^{*}\right|=8$}

The main aim of this section is to find all possible zero divisor graphs of 8 vertices and rings which correspond to them. sequel.

We shall start this section with following lemmas which play a central role in the

\section{Lemma 3.1 :}

Let $\mathrm{R}$ be a ring with $|\mathrm{Z}(\mathrm{R})|^{*}=8$, then $\mathrm{R}$ is local or $\mathrm{R}$ is isomorphic to a product of two local rings.

\section{Proof:}

Since $\left|\mathrm{Z}(\mathrm{R})^{*}\right|=8$, then $\mathrm{R}$ is finite and hence, $\mathrm{R} \cong \mathrm{R}_{1} \times \mathrm{R}_{2} \mathrm{x} \ldots \mathrm{x} \mathrm{R}_{\mathrm{n}}$ where $\mathrm{R}_{\mathrm{i}}$, $\mathrm{i}=1,2, \ldots, \mathrm{n}$ are local rings.

If $\mathrm{n} \geq 4$, then by [5, Lemma 4.7$],\left|\mathrm{Z}(\mathrm{R})^{*}\right| \geq 14$; this is a contradiction. 
Now, consider $n=3$, if $R_{i}$ local but not field for some $1 \leq i \leq 3$, then by Corollary 2.4, $\left|\mathrm{Z}(\mathrm{R})^{*}\right| \geq 13$ which is a contradiction. So $\mathrm{R}_{\mathrm{i}}$ is a field for all $1 \leq \mathrm{i} \leq 3$. Then, by Corollary 2.3

$\left|\mathrm{Z}\left(\mathrm{R}_{1} \mathrm{xR}_{2} \mathrm{xR}_{3}\right)^{*}\right|=\left|\mathrm{R}_{1}\right|\left|\mathrm{R}_{2}\right|+\left|\mathrm{R}_{1}\right|\left|\mathrm{R}_{3}\right|+\left|\mathrm{R}_{2}\right|\left|\mathrm{R}_{3}\right|-\left|\mathrm{R}_{1}\right|-\left|\mathrm{R}_{2}\right|-\left|\mathrm{R}_{3}\right|=8$. If $\left|\mathrm{R}_{1}\right|=\left|\mathrm{R}_{2}\right|=2$ then and $\left|R_{3}\right|=8 / 3$ which is a contradiction. If $\left|R_{i}\right| \geq 3$ for some $i$, then by [5, Lemma 4.5], $\left|Z(R)^{*}\right| \geq 9$ which is a contradiction. Therefore, $\mathrm{n}=1$ or 2 .

\section{Lemma 3.2 :} are fields

Let $\mathrm{R}$ be a ring which is not local and $|\mathrm{Z}(\mathrm{R})|^{*}=8$, then $\mathrm{R} \cong \mathrm{F}_{1} \times F_{2}$, where $\mathrm{F}_{1}$ and $\mathrm{F}_{2}$

\section{Proof:}

Since $\mathrm{R}$ not local, then by Lemma 3.1 $\mathrm{R} \cong \mathrm{R}_{1} \times \mathrm{R}_{2}$, where $\mathrm{R}_{1}, \mathrm{R}_{2}$ are local rings. If $\mathrm{R}_{1}$ and $\mathrm{R}_{2}$ local, but not field, then by [5, Corollary 4.4], $\left|\mathrm{Z}(\mathrm{R})^{*}\right| \geq 11$ which is a contradiction.

If $\mathrm{R}_{1}$ field and $\mathrm{R}_{2}$ local not field, then $\left|\mathrm{m}_{1}\right|=1$. if $\left|\mathrm{m}_{2}\right|=\mathrm{p}$ is prime number, then by [5,Lemma 4.8 ], $\left|R_{2}\right|=p^{2}$ and applied [5,Lemma 4.2], we have $p^{2}+k p-9=0$ where $k=\left|R_{2}\right|-$ 1 , so that $p=\frac{-k+\sqrt{k^{2}+36}}{2} \ldots . .(3)$, since $\mathrm{p}$ is prime, then we have a contradiction. If $\left|\mathrm{m}_{1}\right|$ not prime then $\left|\mathrm{m}_{1}\right| \geq 4$ and since $\left|\mathrm{R}_{2}\right| \geq 2$, then $\left|\mathrm{R}_{1}\right|=9-\left|\mathrm{m}_{1}\right|\left(\left|\mathrm{R}_{2}\right|-1\right) \leq 9-4(2-1)=5$ which is a contradiction. Therefore, $R_{1}$ and $R_{2}$ are fields. Hence, $R \cong F_{1} X F_{2}$, where $F_{1}$ and $F_{2}$ are fields.

\section{Lemma 3.3 :} $\mathrm{Z}_{5} \mathrm{XZ}_{5}$.

Let $\mathrm{R}$ be a ring which is not local and $|\mathrm{Z}(\mathrm{R})|^{*}=8$, then $\mathrm{R} \cong \mathrm{Z}_{2} \times \mathrm{F}_{8}$ or $\mathrm{Z}_{3} \times \mathrm{Z}_{7}$ or

\section{Proof:}

By Lemma3.2 $R \cong F_{1} \times F_{2}$, where $F_{1}, F_{2}$ are fields, we have $\left|F_{1}\right|+\left|F_{2}\right|-2=8$ which implies that $\left|F_{1}\right|+\left|F_{2}\right|=10$, so that $\left|F_{1}\right|=2,\left|F_{2}\right|=8$ or $\left|F_{1}\right|=3,\left|F_{2}\right|=7$ or $\left|F_{1}\right|=5,\left|F_{2}\right|=5$. Therefore, $\mathrm{R} \cong \mathrm{Z}_{2} \mathrm{xF}_{8}$ or $\mathrm{Z}_{3} \mathrm{x} \mathrm{Z}_{7}$ or $\mathrm{Z}_{5} \mathrm{XZ}_{5}$.

Now, we are in a position to give the main result of this section

\section{Theorem 3.4 :}

Let $\mathrm{R}$ be a ring which is not local and $\left|\mathrm{Z}(\mathrm{R})^{*}\right|=8$, then the following graph can be realized as $\Gamma(\mathrm{R})$.

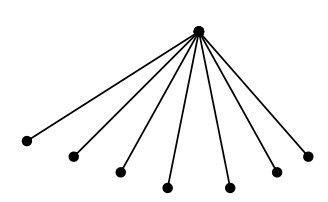

Figure (1)

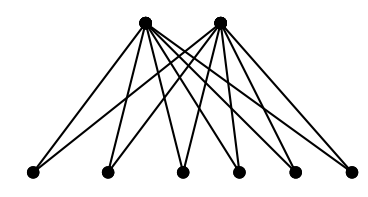

Figure (2)

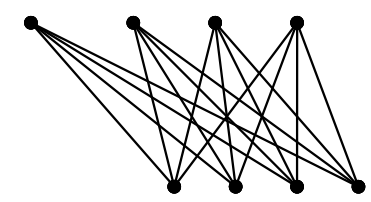

Figure (3),

\section{Proof:}

By Lemma 3.3, then $\mathrm{R} \cong \mathrm{Z}_{2} \times \mathrm{F}_{8}$ or $\mathrm{Z}_{3} \mathrm{XZ}_{7}$ or $\mathrm{Z}_{5} \times \mathrm{Z}_{5}$. In Figure (1), can be realized as $\Gamma\left(Z_{2} x_{8}\right)$. Figure (2), can be realized as $\Gamma\left(Z_{3} \times Z_{7}\right)$. Figure (3), can be realized as $\Gamma\left(\mathrm{Z}_{5} \mathrm{XZ}_{5}\right)$. 


\section{REFERENCES}

[1] D.D. Andersen and P. S. Livingston , (1999), "The zero divisor graph of a commutative ring". Journal of Algebra 217, pp. 434-447

[2] A. Badawi, (2004), "Abstract Algebra Manual: Problems and Solutions 2nd Edition problems and solutions", Nova Science Publishe

[3] I. Beck , (1988), "Coloring of commutative ring". Journal of Algebra 116, pp. 208-226.

[4] G. Carbas and D. Williams, (2000), " Rings of Order p5.I Nonlocal Rings ", Journal of Algebra 231(2), pp. 677-690.

[5] J. T. Wang, (2005), "Zero Divisor of Commutative Rings", M.Sc. Thesis the University of National Chung Cheng, Taiwan. 